\title{
Genome-wide comparison of genes involved in the biosynthesis, metabolism, and signaling of juvenile hormone between silkworm and other insects
}

\author{
Daojun Cheng, Meng Meng, Jian Peng, Wenliang Qian, Lixia Kang and Qingyou Xia \\ State Key Laboratory of Silkworm Genome Biology, Southwest University, Chongqing, China.
}

\begin{abstract}
Juvenile hormone $(\mathrm{JH})$ contributes to the regulation of larval molting and metamorphosis in insects. Herein, we comprehensively identified 55 genes involved in $\mathrm{JH}$ biosynthesis, metabolism and signaling in the silkworm (Bombyx mori) as well as 35 in Drosophila melanogaster, 35 in Anopheles gambiae, 36 in Apis mellifera, 47 in Tribolium castaneum, and 44 in Danaus plexippus. Comparative analysis showed that each gene involved in the early steps of the mevalonate (MVA) pathway, in the neuropeptide regulation of $\mathrm{JH}$ biosynthesis, or in $\mathrm{JH}$ signaling is a single copy in B. mori and other surveyed insects, indicating that these $\mathrm{JH}$-related pathways or steps are likely conserved in all surveyed insects. However, each gene participating in the isoprenoid branch of $\mathrm{JH}$ biosynthesis and $\mathrm{JH}$ metabolism, together with the FPPS genes for catalyzing the final step of the MVA pathway of $\mathrm{JH}$ biosynthesis, exhibited an obvious duplication in Lepidoptera, including B. mori and D. plexippus. Microarray and real-time RT-PCR analysis revealed that different copies of several $\mathrm{JH}$-related genes presented expression changes that correlated with the dynamics of $\mathrm{JH}$ titer during larval growth and metamorphosis. Taken together, the findings suggest that duplication-derived copy variation of $\mathrm{JH}$-related genes might be evolutionarily associated with the variation of $\mathrm{JH}$ types between Lepidoptera and other insect orders. In conclusion, our results provide useful clues for further functional analysis of $\mathrm{JH}$-related genes in $\mathrm{B}$. mori and other insects.
\end{abstract}

Keywords: juvenile hormone, biosynthesis, metabolism, signaling, gene duplication.

Received: November 5, 2013; Accepted: February 27, 2014.

\section{Introduction}

In insects, the sesquiterpenoid juvenile hormone $(\mathrm{JH})$ is synthesized and released from the corpora allata (CA) and cooperates with the steroid 20-hydroxyecdysone (20E) that is synthesized and released from the prothoracic glands to orchestrate insect molting, growth, metamorphosis, via stage-specific changes in the titers of these two endocrine hormones (Dubrovsky, 2005). JH activity is elevated early in each larval instar to maintain larval shape and characteristics, whereas the titer of $20 \mathrm{E}$ is always increased at the end of each larval instar to trigger the transition instar from larva to larva (Bownes and Rembold, 1987; Riddiford, 1994; Wyatt and Davey, 1996; Futahashi and Fujiwara, 2008). In the final larval instar, the JH titer is remarkably decreased and the $20 \mathrm{E}$ titer is increased to a very high level enough to initiate a metamorphic transition from larva to pupa. In addition, JH has been showed to regulate aging and reproduction in insects (Riddiford, 2012; Yamamoto et al., 2013; Zou et al., 2013).

The dynamic change in $\mathrm{JH}$ titer is mainly modulated through biosynthetic and metabolic pathways that are cata-

Send correspondence to Qingyou Xia. State Key Laboratory of Silkworm Genome Biology, Southwest University, No. 1 Tiansheng Road, Beibei District, 400715 Chongqing, China. E-mail: xiaqy@swu.edu.cn. lyzed by different sets of endogenous enzymes (Li et al., 2004; Minakuchi et al., 2006; Noriega et al., 2006; Kinjoh et al., 2007). As summarized in Figure S1, JH biosynthesis involves the mevalonate (MVA) pathway and the isoprenoid branch. The MVA pathway includes eight enzymatic steps and uses acetyl-CoA to generate farnesyl diphosphate (FPP) as a JH precursor. Then, the isoprenoid branch converts the FPP into JH through several continuous steps of oxidization and epoxidation (Kinjoh et al., 2007). In addition, allatotropin (AT) and allatostatin (AS), which belong to the neuropeptide hormone family, promotes and inhibits, respectively, the JH biosynthesis by affecting CA activity (Kataoka et al., 1989; Kramer et al., 1991; Bogus and Scheller, 1996). Furthermore, proper nutritional signals can affect the release of AT and AS by the brain and further results in the activation or inhibition of JH biosynthesis (Noriega, 2004). Moreover, the decrease in JH titer is controlled through its metabolism, which is mainly catalyzed by JH esterase (JHE), JH epoxide hydrolase (JHEH), and JH diol kinase (JHDK). Particularly, several genes involved in JH biosynthesis, metabolism, and signaling have been previously analyzed in insects (Noriega et al., 2006; Kinjoh et al., 2007; Hua-Jun et al., 2011).

Deciphering JH signaling in insects has been attracting increasing attention worldwide. Although the nature of 
JH signaling has not been completely uncovered, some molecules have been demonstrated to be involved in $\mathrm{JH}$ signaling, including $\mathrm{JH}$ binding protein (JHBP), methoprene-tolerant (Met), the 20E receptor EcR, Ultraspiracle (USP), Krüppel homolog 1 (Kr-h1), FKBP39, and Chd64 (Riddiford, 2008; Jindra et al., 2013). Notably, Met protein is a bHLH-PAS transcription factor and has been confirmed as a potential $\mathrm{JH}$ receptor in some insect species (Jindra et al., 2013). In Drosophila melanogaster and Bombyx mori, Met paralog, germ-cell expressed (Gce) or Met2 protein functions as a partner of Met in modulating JH signaling (Abdou et al., 2011; Guo et al., 2012).

Intriguingly, there are eight types of natural JHs characterized in insects, including JH0, JHI, JHII, JHIII, JHIII skipped bisepoxide (JHSB3), JHIII bisepoxide (JHB3), methyl farnesoate (MF), and iso JH0 (Minakuchi et al., 2006; Daimon and Shinoda, 2013). Currently, only JHIII is ubiquitous in all insects. The other four JH variants, including $\mathrm{JH} 0$, iso $\mathrm{JH} 0, \mathrm{JHI}$, and $\mathrm{JHII}$, have been found exclusively in Lepidoptera (Furuta et al., 2013). JHSB3 and JHB3 are specific to Hemiptera and Diptera, respectively. MF is found to be present in both Diptera and Coleoptera. However, little is known about how genes involved in $\mathrm{JH}$ biosynthesis, metabolism, and signaling vary among insects.

As noted above, some of the genes (referred to as JH-related genes) participating in JH-related pathways (biosynthesis, metabolism, and signaling) have been characterized in various insect species. However, with the availability of whole-genome sequence, the number, structure, and evolution of all $\mathrm{JH}$-related genes have not yet been investigated and compared systematically at a genomewide level among insects. The silkworm (B. mori) belongs to the order Lepidoptera. In this study, based on the current B. mori genome assembly (Xia et al., 2008), we performed a genome-wide identification of genes involved in JH biosynthesis, metabolism, and signaling in B. mori; we then evolutionarily compared the $B$. mori genes with their orthologs in other insects.

\section{Materials and Methods}

\section{Gene identification and phylogenetic analysis}

The B. mori genome sequences and the predicted proteins were downloaded from the SilkDB database and were used in our analysis. The predicted protein sets for $D$. melanogaster, Anopheles gambiae, Apis mellifera, Tribolium castaneum, and Danaus plexippus were downloaded from NCBI, Ensembl, or specific databases such as FlyBase for D. melanogaster, BeeBase for A. mellifera (Munoz-Torres et al., 2011), MonarchBase for $D$. plexippus (Zhan and Reppert, 2013), and the Butterfly Genome Database for Heliconius melpomene (Heliconius Genome, 2012).
We used protein sequences or conserved domains of known JH-related genes to query against the predicted protein sets of $B$. mori and of other insects, using the BLAST program with an E-value threshold of less than 1e-6. In addition, an online SMART program was used to search for the functional domains of predicted proteins. Multiple candidate members of a JH-related gene were identified on the basis of agreement with both the E-value threshold and the existence of typical domains. Regarding the nomenclature, candidate genes were defined with an original name if the biological properties (such enzymatic activities) had been confirmed or if the genes had only one copy in each insect surveyed. If a JH-related gene had multiple copies and no functional attributes, we refer them as original-like genes.

The chromosomal distribution of JH-related genes in $B$. mori was determined using a $B$. mori genetics linkage map constructed using single-nucleotide polymorphism (SNP) markers (Yamamoto et al., 2008). The multiple alignments of the complete amino acid sequences or functional domains of JH-related genes were performed using ClustalX (Thompson et al., 1997). Based on the multiple sequence alignment results, neighbor-joining phylogenetic trees for JH-related genes from all surveyed insects were constructed using MEGA4.0 (Tamura et al., 2007) with a bootstrap of 1000 replicates.

\section{Microarray-based gene expression analysis}

The spatio-temporal expression patterns of JHrelated genes in $B$. mori were first surveyed using the microarray method. Microarray gene expression data of multiple larval tissues from $B$. mori larvae at the third day of the fifth instar and related analytical methods from our previous report (Xia et al., 2007) were used to profile the tissue-specific expression of JH-related genes. In nine larval tissues, including the $\mathrm{A} / \mathrm{MSG}$ (anterior/median silk gland), PSG (posterior silk gland), testis, ovary, fat body, midgut, integument, hemocyte, Malpighian tubule, and head (containing the brain and the associated glands in the retrocerebral complex, corpora allata (CA), corpus cardiacum (CC)), a total of 10,393 genes (transcripts) have been estimated to be activated based on an intensity threshold of 400 (Xia et al., 2007). We retrieved the microarray data for $B$. mori JH-related genes from the active gene selection to examine their tissue expression profiles.

The developmental expression pattern was analyzed using microarray data of $B$. mori gene expression during metamorphosis (unpublished data), including 19 developmental time points during the larva-pupa-adult transitions, namely, V4 (fourth day of the fifth larval instar), V5, V6, V7, W0 (beginning of wandering), W12 (12 hours after wandering), W24, W36, W48 (completing spinning), W60 (immediately after pupation), W72, W96, W120, W144, W168, W192, W216, W240, and adulthood. Gene expression in $B$. mori larvae at V3 (third day of the fifth larval instar) was set as the common reference. The ratio between 
the experimental and reference intensities for a JH-related gene was used to evaluate expression changes during the larva-pupa-adult transitions of $B$. mori. The related analytical method was based on our previous reports (Xia et al., 2007; Huang et al., 2009). Tissue and developmental expression patterns from the microarray analysis were visualized using the GeneCluster2.0 program (Reich et al., 2004).

\section{Real-time quantitative RT-PCR examination of gene expression}

We also used a real-time quantitative RT-PCR approach to examine expression patterns of JH-related genes during the larval growth of $B$. mori. The $B$. mori strain Dazao was reared under a temperature of $25^{\circ}$ C. B. mori larvae were collected at ten time points during the larval feeding and molting stages from the fourth instar to the fifth instar, including IV0 ( 0 hour after the third larval molt, namely, the beginning of the fourth instar), IV1 (day 1 after the third molt), IV2 (day 2 after the third molt), IV3 (day 3 after the third molt), IV4 (day 4 after the third molt), IVM (just initiating the fourth larval molt), V0 ( 0 hours after the fourth molt, namely, the beginning of the fifth instar), and V1 (day 1 of the fifth instar).

Total RNA was extracted using TRIzol reagent (Invitrogen, USA) and was reverse transcribed into cDNA with M-MLV reverse transcriptase (Promega, USA). Real-time RT-PCR was conducted as described in our previous study (Wang et al., 2008). The B. mori ribosomal protein L3 $(R p L 3)$ was used as an internal control. All primers used are listed in Table S1.

\section{Results}

\section{Inventory of genes involved in $\mathrm{JH}$ biosynthesis, metabolism, and signaling in $B$. mori and other insects}

We used the amino acid sequences of known insect $\mathrm{JH}$-related genes to search against the predicted B. mori proteins using the BLAST program. Initially, $55 \mathrm{JH}$-related genes were identified, including 34 for JH biosynthesis, 13 for $\mathrm{JH}$ metabolism, and eight for $\mathrm{JH}$ signaling (Table 1). Several JH-related genes exhibited multiple copies, including farnesyl diphosphate synthase (FPPS), farnesyl

Table 1 - Inventory of JH-related genes in the B. mori genome.

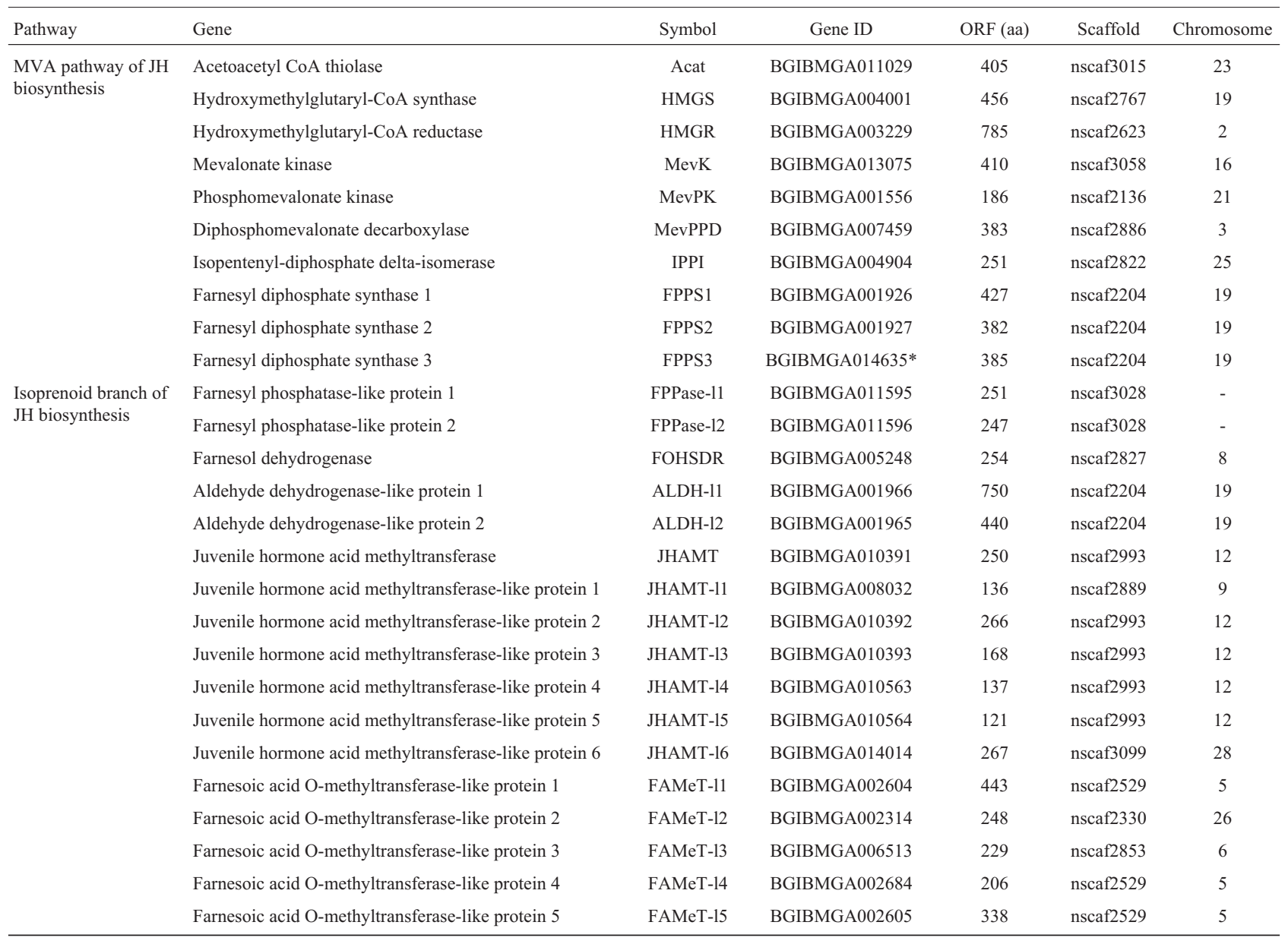


Table 1 (cont.)

\begin{tabular}{|c|c|c|c|c|c|c|}
\hline Pathway & Gene & Symbol & Gene ID & ORF (aa) & Scaffold & Chromosome \\
\hline \multirow{6}{*}{$\begin{array}{l}\text { Neuropeptide regula- } \\
\text { tion of JH } \\
\text { biosynthesis }\end{array}$} & Farnesoic acid O-methyltransferase-like protein 6 & FAMeT-16 & BGIBMGA006319 & 224 & nscaf 2852 & 6 \\
\hline & Farnesoic acid O-methyltransferase-like protein 7 & FAMeT-17 & BGIBMGA006318 & 829 & nscaf2852 & 6 \\
\hline & Cytochrome P450 15C1 & Cyp 15 C1 & BGIBMGA011708 & 288 & nscaf3031 & 11 \\
\hline & Allototropin & AT & BGIBMGA011850 & 291 & nscaf3031 & 11 \\
\hline & Allatostatin & AS & BGIBMGA014377 & 150 & scaffold416 & 14 \\
\hline & Allatostatin receptor & ASR & BGIBMGA005708 & 362 & nscaf 2830 & - \\
\hline \multirow[t]{13}{*}{ JH metabolism } & Juvenile hormone esterase & JHE & BGIBMGA000772 & 567 & nscaf1705 & 25 \\
\hline & Juvenile hormone esterase-like protein 1 & JHE-11 & BGIBMGA000774 & 566 & nscaf1705 & 25 \\
\hline & Juvenile hormone esterase-like protein 2 & JHE-12 & BGIBMGA000775 & 560 & nscaf1705 & 25 \\
\hline & Juvenile hormone esterase-like protein 3 & JHE-13 & BGIBMGA000776 & 572 & nscaf1705 & 25 \\
\hline & Juvenile hormone epoxide hydrolase & JHEH & BGIBMGA013930 & 461 & nscaf3099 & 28 \\
\hline & Juvenile hormone epoxide hydrolase-like protein 1 & JHEH-11 & BGIBMGA011468 & 401 & nscaf3027 & 23 \\
\hline & Juvenile hormone epoxide hydrolase-like protein 2 & JHEH-12 & BGIBMGA009211 & 510 & nscaf2943 & 14 \\
\hline & Juvenile hormone epoxide hydrolase-like protein 3 & JHEH-13 & BGIBMGA013994 & 637 & nscaf3099 & 28 \\
\hline & Juvenile hormone epoxide hydrolase-like protein 4 & JHEH-14 & BGIBMGA013793 & 395 & nscaf3097 & 28 \\
\hline & Juvenile hormone epoxide hydrolase-like protein 5 & JHEH-15 & BGIBMGA013929 & 355 & nscaf3099 & 28 \\
\hline & Juvenile hormone diol kinase & JHDK & BGIBMGA008814 & 183 & nscaf2925 & 3 \\
\hline & Juvenile hormone diol kinase-like protein 1 & JHDK-11 & BGIBMGA008813 & 182 & nscaf2925 & 3 \\
\hline & Juvenile hormone diol kinase-like protein 2 & JHDK-12 & BGIBMGA008815 & 179 & nscaf 2925 & 3 \\
\hline \multirow[t]{6}{*}{ JH signaling } & Juvenile hormone binding protein & JHBP & BGIBMGA011549 & 243 & nscaf3027 & 23 \\
\hline & FKBP39 & FKBP39 & BGIBMGA001490 & 402 & nscaf 2136 & 21 \\
\hline & Chd64 & Chd64 & BGIBMGA007092 & 174 & nscaf 2865 & 17 \\
\hline & Methoprene-tolerant 1 & Met1 & BGIBMGA005416 & 455 & nscaf2828 & 8 \\
\hline & Ultraspiracle & USP & BGIBMGA006183 & 270 & nscaf2847 & 4 \\
\hline & Kruppel homolog 1 & Kr-h1 & BGIBMGA003160 & 348 & nscaf2589 & 4 \\
\hline
\end{tabular}

Note: * indicates new gene assembly. - indicates unknown.

phosphatase (FPPase), aldehyde dehydrogenase (ALDH), JH acid methyltransferase (JHAMT), farnesoic acid $O$-methyltransferase (FAMeT), JHE, JHEH, and JHDK. All JH-related genes for $B$. mori mapped to different chromosomes except for the FPPase gene and allatostatin receptor $(A S R)$ gene (Figure 1 and Table 1). Intriguingly, the copies of several JH-related gene with multiple copies were distributed on the same chromosome in a tandem manner, for example, FPPS on chromosome 12, JHDK on chromosome 3, FAMeT on chromosomes 5 or 6 , JHAMT on chromosome 12, JHE on chromosome 25, and JHEH on chromosome 28.

To determine comprehensively the evolution of $\mathrm{JH}$ related genes among insects, we further identified JH-related genes in other insects, namely, 35 in D. melanogaster, 35 in A. gambiae, 36 in A. mellifera, 47 in T. castaneum, and 44 in D. plexippus (Table 2, Table S2). Some JHrelated genes in these five insects had at least two copies, including three genes (Farnesol dehydrogenase (FOHSDR), JHE, and JHEH) in D. melanogaster, three (FOHSDR, JHE, and JHEH) in A. gambiae, four (FPPase,
FOHSDR, FAMeT, and JHE) in A. mellifera, five (FPPase, FOHSDR, ALDH, JHAMT, and JHEH) in T. castaneum, and six (FPPS, FPPase, ALDH, FAMeT, JHEH, and $J H D K)$ in D. plexippus. Moreover, the copies of several $\mathrm{JH}$-related genes in these insects were distributed in tandem on a chromosome, including the JHEHs in $D$. melanogaster; the JHEHs and FOHSDRs in A. gambiae; the JHAMTs and JHEHs in T. castaneum; and the FPPSs, FPPases, FAMeTs, JHEHs, and JHDKs in D. plexippus. On the basis of these and similar observations in B. mori, we speculate that the JH-related genes with multiple copies were duplicated during the evolution of $B$. mori and other insects.

\section{The MVA pathway of $\mathrm{JH}$ biosynthesis}

The upstream mevalonate (MVA) pathway of JH biosynthesis is responsible for producing the $\mathrm{JH}$ precursor farnesyl diphosphate (FPP). As shown in Figure S1, the MVA pathway involved eight enzymatic steps. Interestingly, each of the enzyme-encoding genes involved in the first seven steps of the MVA pathway, which produce the 

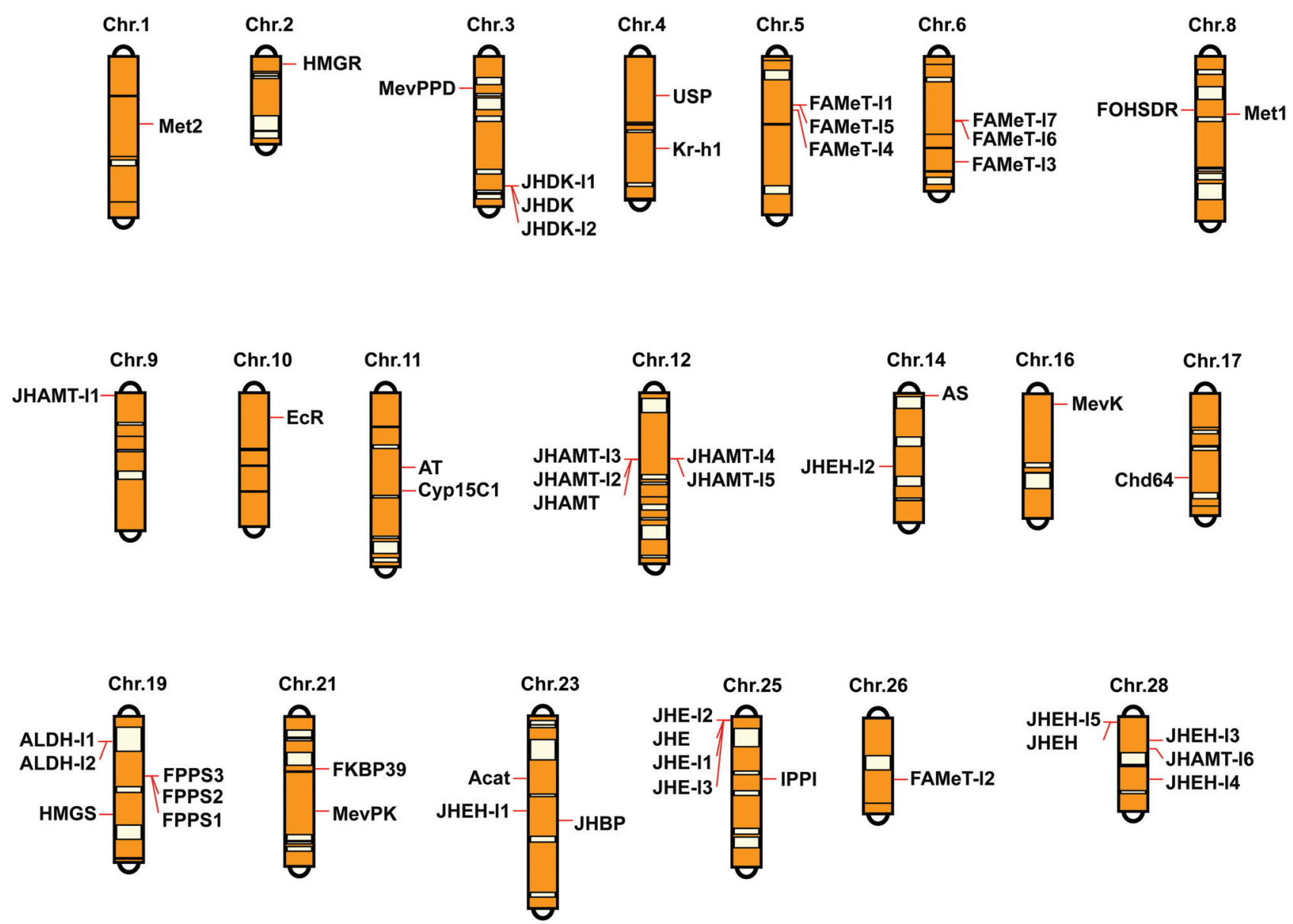

Figure 1 - Chromosomal distribution of JH-related genes in B. mori. Based on the assembly of the whole-genome sequence and single-nucleotide polymorphism (SNP) markers linkage map for B. mori, a total of $52 \mathrm{JH}$-related genes were mapped on the different chromosomes of $B$. mori. Several different copies of each of the JH-related genes with multiple copies are clustered in tandem on the chromosomes.

FPP precursor isopentenyl diphosphate (IPP), existed as a single copy in B. mori and five other surveyed insects and showed a 1:1:1:1:1:1 orthologous relationship (Table 2).

Farnesyl diphosphate synthase (FPPS) catalyzes the final reaction of the MVA pathway, converting IPP into the JH precursor FPP. Three transcripts of the FPPS gene have been identified in B. mori (Kinjoh et al., 2007). We noted that only two FPPS transcripts matched well with two predicted genes for B. mori, namely, FPPS1 for BGIBMGA001926 and FPPS2 for BGIBMGA001927. We further used the complete cDNA sequence of the FPPS3 transcript to search against the $B$. mori genome assembly using the BLASTn algorithm. In this search, we found that the FPPS3 transcript matched the downstream genomic region of the FPPS2 gene; therefore, we defined FPPS3 as BGIBMGA014635 for a supplement of the B. mori predicted genes. Strikingly, a comparative analysis revealed a copy number variation in the FPPS genes among the surveyed insects. In addition to three copies in B. mori, there were six copies in A. mellifera, two in D. plexippus, and one each in D. melanogaster, $A$. gambiae, and T. castaneum. From the phylogenetic tree of the FPPS and FPPS-like genes that were identified here and collected from online resources for other insects (Figure 2), we observed that all FPPS and FPPS-like genes from $B$. mori and other lepidopterans, including $D$. plexippus, Heliconius melpomene, Choristoneura fumiferana, Mythimna unipuncta, and Agrotis ipsilon, clustered into two groups, and different copies from each insect were separately grouped, indicating that the FPPS duplication in lepidopterans may have occurred after their separation from other insect species and before their separation from each other. However, all six FPPS-like genes in A. mellifera grouped well together, suggesting that the FPPS genes from A. mellifera may have been duplicated after the separation of A. mellifera from other insects. The FPPS genes from other five insect species, including Aedes aegypti, Culex quinquefasciatus, Anthonomus grandis, Dendroctonus jeffreyi, and Nasonia vitripennis, also existed as a single copy and clustered together with the FPPS genes from $D$. melanogaster, A. gambiae, and T. castaneum.

\section{The isoprenoid branch of $\mathrm{JH}$ biosynthesis}

The downstream isoprenoid branch pathway of $\mathrm{JH}$ biosynthesis converts FPP to JH (Minakuchi et al., 2006). 
Table 2 - Copy number of JH-related genes in B. mori and the other insects.

\begin{tabular}{|c|c|c|c|c|c|c|c|c|}
\hline Pathway & Gene & Symbol & $\begin{array}{c}\text { B. mori } \\
\text { (Lepidoptera) }\end{array}$ & $\begin{array}{l}\text { D. melanogaster } \\
\text { (Diptera) }\end{array}$ & $\begin{array}{l}\text { A. gambiae } \\
\text { (Diptera) }\end{array}$ & $\begin{array}{l}\text { A. mellifera } \\
\text { (Hymenoptera) }\end{array}$ & $\begin{array}{l}\text { T. castaneum } \\
\text { (Coleoptera) }\end{array}$ & $\begin{array}{l}\text { D. plexippus } \\
\text { (Lepidoptera) }\end{array}$ \\
\hline \multirow{8}{*}{$\begin{array}{l}\text { MVA pathway } \\
\text { of JH } \\
\text { biosynthesis }\end{array}$} & Acetoacetyl CoA thiolase & ACAT & 1 & 1 & 1 & 1 & 1 & 1 \\
\hline & $\begin{array}{l}\text { Hydroxymethylglutaryl-CoA } \\
\text { synthase }\end{array}$ & HMGS & 1 & 1 & 1 & 1 & 1 & 1 \\
\hline & $\begin{array}{l}\text { Hydroxymethylglutaryl-CoA } \\
\text { reductase }\end{array}$ & HMGR & 1 & 1 & 1 & 1 & 1 & 1 \\
\hline & Mevalonate kinase & MevK & 1 & 1 & 1 & 1 & 1 & 1 \\
\hline & Phosphomevalonate kinase & MevPK & 1 & 1 & 1 & 1 & 1 & 1 \\
\hline & $\begin{array}{l}\text { Diphosphomevalonate decar- } \\
\text { boxylase }\end{array}$ & MevPPD & 1 & 1 & 1 & 1 & 1 & 1 \\
\hline & $\begin{array}{l}\text { Isopentenyl-diphosphate } \\
\text { delta-isomerase }\end{array}$ & IPPI & 1 & 1 & 1 & 1 & 1 & 1 \\
\hline & Farnesyl diphosphate synthase & FPPS & 3 & 1 & 1 & 6 & 1 & 2 \\
\hline \multirow{7}{*}{$\begin{array}{l}\text { Isoprenoid } \\
\text { branch of JH } \\
\text { biosynthesis }\end{array}$} & Farnesyl phosphatase & FPPase & 2 & 1 & 1 & 2 & 3 & 2 \\
\hline & Farnesol dehydrogenase & FOHSDR & 1 & 6 & 6 & 2 & 7 & 1 \\
\hline & Aldehyde dehydrogenase & ALDH & 2 & 1 & 1 & 1 & 6 & 2 \\
\hline & $\begin{array}{l}\text { Juvenile hormone acid } \\
\text { methyltransferase }\end{array}$ & JHAMT & 7 & 1 & 1 & 1 & 3 & 1 \\
\hline & $\begin{array}{l}\text { Farnesoic acid } \\
\text { O-methyltransferase }\end{array}$ & FAMeT & 7 & 1 & 1 & 2 & 1 & 5 \\
\hline & Cytochrome P450 15A1 & Cyp15A1 & - & - & - & 1 & 1 & - \\
\hline & Cytochrome P450 15C1 & Cyp15C1 & 1 & - & - & - & - & 1 \\
\hline \multirow{4}{*}{$\begin{array}{l}\text { Neuropeptide } \\
\text { regulation of JH } \\
\text { biosynthesis }\end{array}$} & Allototropin & AT & 1 & - & 1 & - & 1 & 1 \\
\hline & Allatostatin & AS & 1 & 1 & 1 & 1 & 1 & 1 \\
\hline & Allototropin receptor & ATR & 1 & 1 & 1 & 1 & 1 & 1 \\
\hline & Allatostatin receptor & ASR & 1 & 1 & 1 & 1 & 1 & 1 \\
\hline \multirow[t]{3}{*}{ JH metabolism } & Juvenile hormone esterase & JHE & 4 & 2 & 2 & 2 & 1 & 1 \\
\hline & $\begin{array}{l}\text { Juvenile hormone epoxide } \\
\text { hydrolase }\end{array}$ & JHEH & 6 & 3 & 3 & 1 & 5 & 8 \\
\hline & Juvenile hormone diol kinase & JHDK & 3 & 1 & 1 & 1 & 1 & 2 \\
\hline \multirow[t]{8}{*}{ JH signaling } & $\begin{array}{l}\text { Juvenile hormone binding } \\
\text { protein }\end{array}$ & JHBP & 1 & 1 & 1 & 1 & 1 & 1 \\
\hline & FKBP39 & FKBP39 & 1 & 1 & 1 & 1 & 1 & 1 \\
\hline & Chd64 & Chd64 & 1 & 1 & 1 & 1 & 1 & 1 \\
\hline & $\begin{array}{l}\text { Methoprene-tolerant/Methopr } \\
\text { ene-tolerant } 1\end{array}$ & Met/Met1 & 1 & 1 & 1 & 1 & 1 & 1 \\
\hline & $\begin{array}{l}\text { Germ cell ex- } \\
\text { pressed/Methoprene-tolerant } 2\end{array}$ & Gce/Met2 & 1 & 1 & - & - & - & 1 \\
\hline & Ecdysone receptor & $\mathrm{EcR}$ & 1 & 1 & 1 & 1 & 1 & 1 \\
\hline & Ultraspiracle & USP & 1 & 1 & 1 & 1 & 1 & 1 \\
\hline & Kruppel homolog 1 & Kr-h1 & 1 & 1 & 1 & 1 & 1 & 1 \\
\hline
\end{tabular}

Note: - represents no identification.

Recently, in insects, three types of catalytic enzymes, including farnesyl pyrophosphate phosphatase (FPPase), farnesol dehydrogenase (FOHSDR), and aldehyde dehydrogenase (ALDH), have been successfully identified as being responsible for the sequential conversion of FPP into farnesol, farnesal, and farnesoic acid (FA) (Mayoral et al., 2009; Nyati et al., 2013; Rivera-Perez et al., 2013). In addition to the previous prediction of these genes in several insects, we identified two FPPase-like genes, one FOHSDR gene, and two $A L D H$-like genes in D. plexippus. Although each of these genes exhibited a different number of copies among the surveyed insects, the copy number of each gene was the same in B. mori and D. plexippus as well as in $D$. melanogaster and A. gambiae (Table 2 and Table S2).

The conversion of FA into JH is completed via two catalytic reactions in insects, namely, epoxidation and methyl esterification. Notably, this conversion can occur in two ways (Figure S1). One involves FA oxidation by Cyp15C1 to form JH acid (JHA), after which JHA is methylated by juvenile hormone acid methyltransferase (JHAMT) to synthesize JH. Another way is that FA is methylated to form methyl farnesoate (MF) by farnesoic 


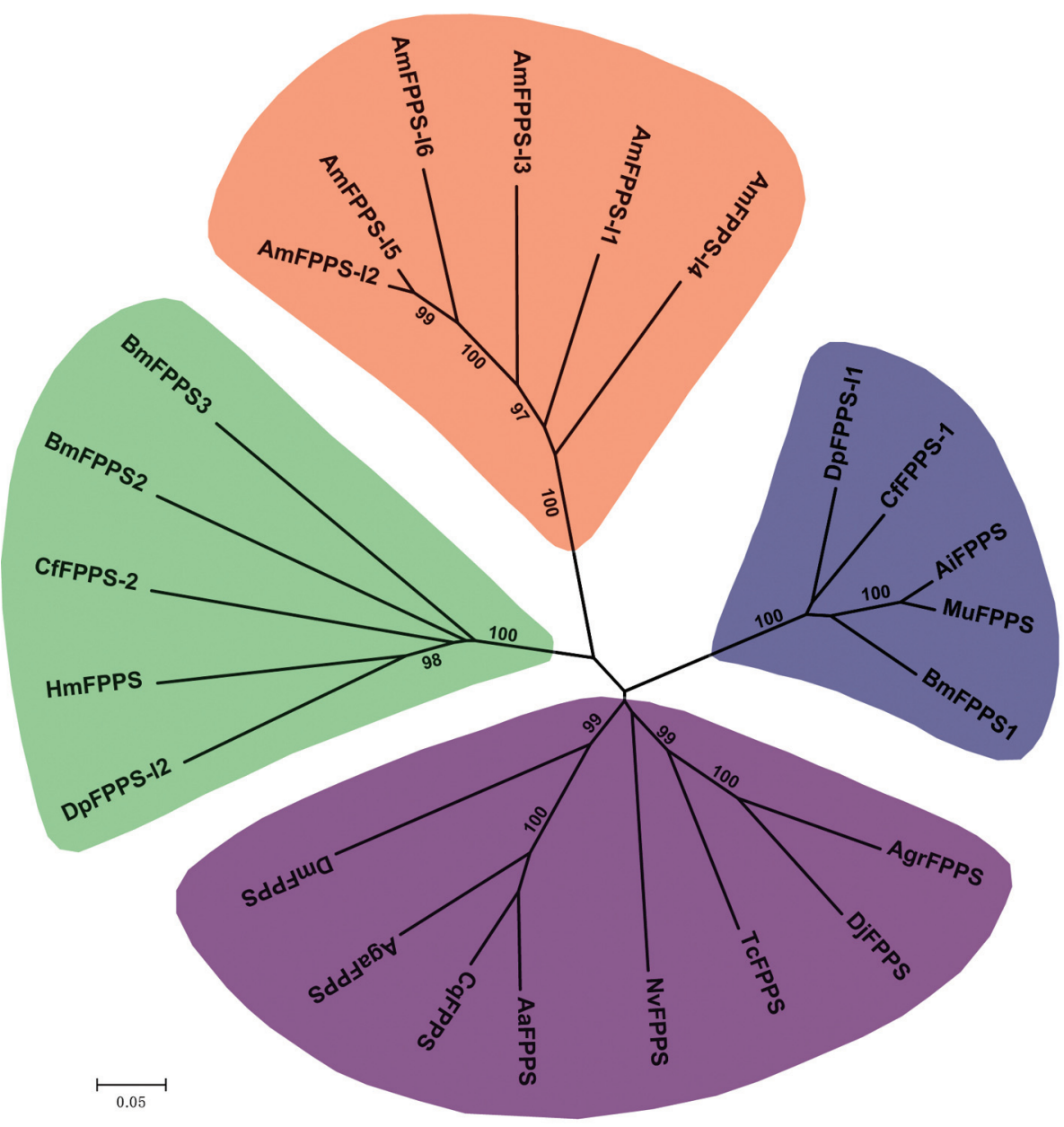

Figure 2 - Phylogenetic tree of the FPPS genes from B. mori and other insects. Based on the multiple alignments of the amino acid sequences of FPPS proteins from $B$. mori and other insects, a phylogenetic tree was constructed using the neighbor-joining method with 1000bootstrap replicates after removing the highly divergent sequences at the $\mathrm{N}$ - or C-terminus. Bootstrap values $>60 \%$ are marked. Bm, Bombyx mori; Dm, Drosophila melanogaster; Aga, Anopheles gambiae; Am, Apis mellifera; Tc, Tribolium castaneum; Dp, Danaus plexippus; Cf, Choristoneura fumiferana; Hm, Heliconius melpomene; Mu, Mythimna unipuncta; Agi, Agrotis ipsilon; Aa, Aedes aegypti; Cq, Culex quinquefasciatus; Agr, Anthonomus grandis; Dj, Dendroctonus jeffreyi; Nv, Nasonia vitripennis.

acid O-methyltransferase (FAMeT), after which MF is oxidized by Cyp15A1 to produce $\mathrm{JH}$.

Cyp15C1 and Cyp15A1, the two genes involved in FA epoxidation, belong to the Cyp15 subfamily of cytochrome $\mathrm{P} 450$ enzymes. The Cyp $15 \mathrm{Cl}$ gene has been identified in B. mori and D. plexippus, and the Cyp15A1 gene has been characterized in $T$. castaneum and A. mellifera (Daimon et al., 2012; Daimon and Shinoda 2013). No Cyp15C1 or Cyp15A1 was identified in D. melanogaster. We performed a BLASTp search against the genome of $H$. melpomene (Lepidoptera) and identified a homolog of the B. mori Cyp $15 C 1$ gene, namely, HMEL006305, further indicating that Cyp15C1 may be Lepidoptera-specific.

JHAMT and FAMeT are considered two catalytic enzymes for the methyl esterification of FA. One authentic JHAMT enzyme was first characterized in B. mori (Shinoda and Itoyama, 2003). As listed in Table 1, the gene (BGIBMGA010391) encoding the authentic JHAMT and six JHAMT-like genes (from JHAMT-ll to JHAMT-l6) were found in the $B$. mori genome, and each contains a methyltransf_12 domain. Furthermore, three JHAMT copies (including authentic JHAMT, JHAMT-l1, and JHAMT12) were predicted in T. castaneum, whereas only one copy of JHAMT gene was identified in four other insects (Table 2 and Table S2). A phylogenetic tree revealed that the different copies of the JHAMT gene in B. mori and D. plexippus grouped well together, and that the three JHAMT copies in T. castaneum also grouped into a clade (Figure S2), suggesting that the JHAMT gene in B. mori or T. castaneum was duplicated after their separation from other insect species.

FAMeT has been found to exist in insects and crustaceans (Hui et al., 2010), and evidence from $D$. melanogaster has suggested that FAMeT has a minor role in $\mathrm{JH}$ biosynthesis, but it may play a major role in JH signaling (Burtenshaw et al., 2008; Zhang et al., 2010). Our data revealed that in the $B$. mori genome, there are seven FAMeT-like genes, each of which contains two typical 
Methyltransf_FA and DM9 domains. Comparatively, FAMeT exists as a single copy in T. castaneum as well as in two Diptera insects but as two copies in A. mellifera and five copies in D. plexippus (Table 2 and Table S2). A phylogenetic tree of insect FAMeT or FAMeT-like genes was constructed using their coding sequences containing two functional domains (Figure S2). In this tree, with the exception of $A$. mellifera, one copy of the FAMeT gene from each of the five remaining insects grouped into a clade. Other copies of the FAMeT gene from B. mori, A. mellifera, and $D$. plexippus grouped together into an additional clade in an irregular manner. This phylogenetic relationship indicates that the duplication of the different $F A M e T$ copies may have occurred after insect radiation and subsequently undergone a rapid sequence diversification during insect evolution.

\section{Neuropeptide regulation of $\mathrm{JH}$ biosynthesis}

Insect $\mathrm{JH}$ biosynthesis is also modulated by two neuropeptides, namely, allatotropin (AT) and allatostatin (AS). AT and AS play antagonistic roles during JH biosynthesis, with the former being a stimulator and the later being an inhibitor of CA activity (Stay, 2000). As listed in Table 1, from the B. mori genome, we retrieved four genes that respectively encode AT, AS, the AT receptor (ATR), and the AS receptor (ASR), which have been previously reported in other insects (Secher et al., 2001; Park et al., 2002; Roller et al., 2008; Yamanaka et al., 2008; Horodyski et al., 2011). In addition, $A S, A S R$, and $A T R$ were all identified in five other insects, whereas $A T$ gene was predicted only in A. gambiae, T. castaneum, and $D$. plexippus in our analysis. Notably, each of the identified genes related to the neuropeptide regulation of JH biosynthesis appears to exist as a single copy in B. mori and the other surveyed insects (Table 2).

\section{Enzymes involved in $\mathrm{JH}$ metabolism}

The metabolic degradation of JH contributes to the reduction of $\mathrm{JH}$ titer and is catalyzed by three enzymes, namely, JHE, JHEH, and JHDK. As shown in Figure S1, JHE catalyzes the conversion of JH into $\mathrm{JH}$ acid (JHa) or the conversion of $\mathrm{JH}$ diol (JHd) into $\mathrm{JH}$ acid diol (JHad). A previous report characterized one $J H E$ gene encoding an authentic JHE enzyme in B. mori (Hirai et al., 2002). Here, in addition to the known $J H E$ gene, three $J H E$-like genes (namely, JHE-l1, JHE-l2, and JHE-l3) were identified from the $B$. mori genome (Table 1). Moreover, we retrieved different copies of the $J H E$ gene in five other insects, namely, 2, 2, 2, 1, and 1 for D. melanogaster, A. gambiae, A. mellifera, T. castaneum, and D. plexippus, respectively (Table 2 and Table S2). All the identified JHE genes contain a COesterase domain. A phylogenetic analysis showed that the $J H E$ genes in Lepidoptera, Hymenoptera, Diptera, or Coleoptera separately grouped well together (Figure S3), consistent with the classical phylogeny of these insect spe- cies. Notably, different copies of the JHE gene in $D$. melanogaster grouped first together, as did those in $A$. gambiae and A. mellifera, suggesting that JHE duplication in these three species occurred after their separation. Nevertheless, in Lepidoptera, JHE from B. mori grouped first with $J H E$ from $D$. plexippus and then with the grouping clade of JHE-l1 and JHE-l2 from B. mori, suggesting that $J H E$ in both $B$. mori and D. plexippus may have a common ancestor, and the other three $J H E$-like genes in B. mori may have undergone a great sequence diversification after their duplication from $J H E$.

JHEH catalyzes the conversion of JH into JHa or the conversion of JHa into JHad. According to previous reports in B. mori (Zhang et al., 2005; Seino et al., 2010), in addition to an authentic $J H E H$, there are five $J H E H$-like genes in the B. mori genome (Table 1). The copy numbers of the $J H E H$ genes vary, with three in D. melanogaster, three in A. gambiae, one in A. mellifera, five in T. castaneum, and seven in D. plexippus (Table 2 and Table S2). On the phylogenetic tree of the $J H E H$ genes (Figure S3), different copies of the $J H E H$ gene from each of four insect species $(D$. melanogaster, A. gambiae, A. mellifera, and T. castaneum) grouped first together, indicating that the $J H E H$ gene from these four species may have undergone species-specific duplication after their separation from the other insects. Moreover, the copies of the $J H E H$ genes from two lepidopterans, B. mori and D. plexippus, grouped into four clades, and each clade contained different copies from both of these species, suggesting that $J H E H$ duplication in Lepidoptera may have occurred before their radiation.

JHDK is required for the conversion of JHd into JH diol phosphate (JHdp). To date, JHDK has been functionally characterized in two Lepidoptera insects, namely, $B$. mori and Manduca sexta (Maxwell et al., 2002; Li et al., 2005). Here, three copies of the JHDK gene were identified in the B. mori genome (Table 1), including one authentic $J H D K$ and two $J H D K$-like genes, each of which contains an EF-hand domain. Furthermore, as listed in Table 2 and Table $\mathrm{S} 2$, there were two copies of the JHDK gene in another lepidopteran, D. plexippus, but only one copy was identified in four other surveyed insects. Phylogenetic analysis showed that the $J H D K$ copies from Lepidoptera grouped into two clades, and the $J H D K$ genes from other insects grouped well together (Figure S3), suggesting that JHDK duplication in Lepidoptera may have also occurred before their separation.

\section{Genes involved in the JH signaling pathway}

Recently, additional evidence has shown that the bHLH-PAS transcription factor Met is a potential receptor for JH signaling (Jindra et al., 2013). Met and its paralogous gene Gce were first identified in D. melanogaster (Wilson and Fabian, 1986; Baumann et al., 2010b), in which they were partially redundant in mediating $\mathrm{JH}$ signaling (Baumann et al., 2010a; Abdou et al., 2011). Two copies of the 
Met gene, Met1 and Met2, have been characterized in $B$. mori (Li et al., 2010; Guo et al., 2012; Kayukawa et al., 2012). Intriguingly, our analysis also identified two copies of the Met gene in two other lepidopteran insects, $D$. plexippus and $H$. melpomene, namely DpMet-l1 and DpMet-l2 in D. plexippus (Table 2 and Table S2) and HmMet-l1 (HMEL011931) and HmMet-l1 (HMEL009818) in H. melpomene. However, only one copy of the Met gene was identified in A. gambiae, A. mellifera, and $T$. castaneum (Table 2 and Table S2). Phylogenetic analysis revealed that Met and Gce from D. melanogaster grouped together (Figure 3). In Lepidoptera, Met1 and Met-l1 grouped into one clade, whereas Met 2 and Met-l2 grouped into another, indicating that the Met duplication in Lepidoptera may have occurred before their separation.

In addition, some molecules, including JHBP, FKBP39, Chd64, EcR, USP, and Kr-h1, have been demonstrated to be involved in JH signaling (Dubrovsky, 2005; Li et al., 2007; Jindra et al., 2013). Our results showed that each of these six genes was identified as having one copy in B. mori and five other insect species (Table 2 and Table S2).

\section{Expression profiles of $\mathrm{JH}$-related genes in multiple tissues of $B$. mori larvae}

Using microarray data of gene expression in multiple tissues of B. mori larvae on the third day of the fifth instar when JH titer is present (Sakurai and Niimi, 1997; Xia et

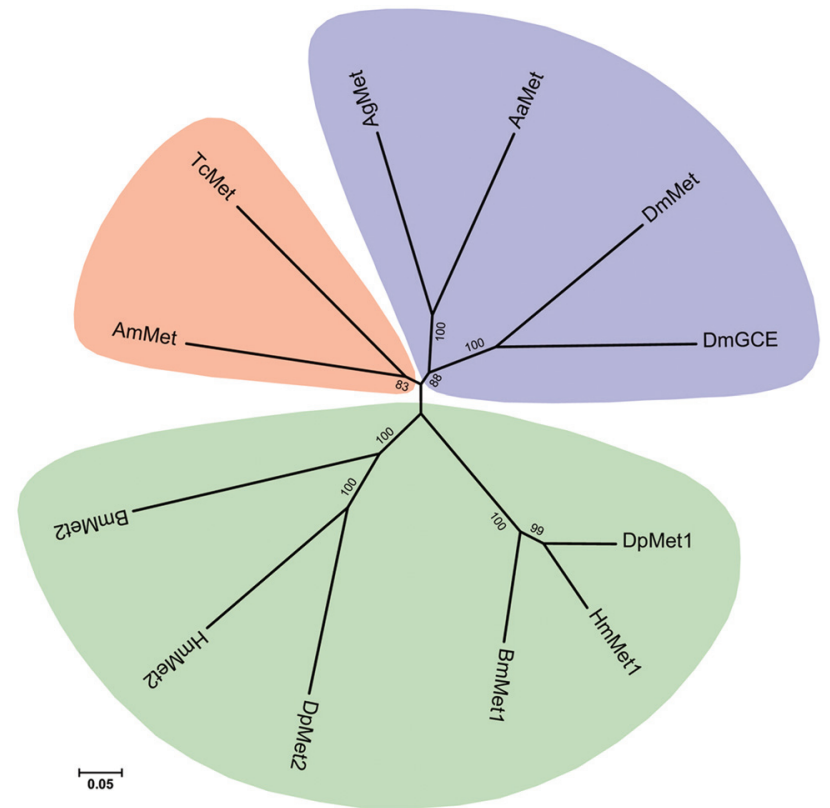

Figure 3 - Phylogenetic tree of the Met genes in B. mori and other insects. The amino acid sequences encoding by Met genes from seven insect species, including Bombyx mori (Bm), Drosophila melanogaster (Dm), Anopheles gambiae (Aga), Apis mellifera (Am), Tribolium castaneum (Tc), Danaus plexippus (Dp), and Heliconius melpomene (Hm), were used to build a phylogenetic tree. See Figure 2 for a detailed description of the approaches for constructing phylogenetic tree. al., 2007), we investigated the tissue expression of JHrelated genes. As a result, 36 of $55 \mathrm{JH}$-related genes were detected in at least one tissue (Figure 4). Among JH biosynthesis-related genes, Acat, MevPK, IPPI, FPPS2, ALDH-l1, JHAMT-I6, FAMeT-I4, and Cyp15C1 were expressed in the head (mainly containing $\mathrm{CA}, \mathrm{CC}$, and brain), which may be directly involved in JH biosynthesis. In particular, several JH biosynthesis-related genes showed either high expression in other larval tissues or ubiquitous expression, such as Acat and MevPK in all surveyed tissues; FAMeT-l1, FAMeT-15, and FAMeT-l7 in the midgut; and JHAMT-l4, JHAMT-l5, and JHAMT-l6 in the fat body and integument. This indicates that these genes may function in other biological processes.

Some genes involved in JH metabolism are mostly expressed in the midgut or Malpighian tubule (Figure 4). For example, five genes, namely, JHE-l1, JHEH, JHEH-l4, $J H D K$, and $J H D K-l 1$, were expressed in both the midgut and Malpighian tubule. JHEH-l1 and JHDK-l2 were expressed in the midgut and Malpighian tubule, respectively. $J H E-l 3$ and JHEH-l2 expression was enriched in both the A/MSG and PSG, whereas JHEH6 was highly expressed in all tissues with the exception of hemocytes. JHEH-l2 was specifically expressed in the A/MSG. In addition, among the genes involved in the JH signaling pathway, JHBP, Met 1, EcR, FKBP39, and Chd64 were all weakly expressed in at least one larval tissue. In particular, FKBP39 and Chd64 were expressed in all analyzed tissues. However, from the microarray data, the expression of three genes, USP, Met2, and $K r-h 1$, was not observed in any tissue. It is possible that the expression levels for these three transcription factors may be too low at day 3 of the fifth larval instar and were therefore difficult to be detected.

\section{Expression profiles of $\mathrm{JH}$-related genes during $B$. mori metamorphosis}

Based on microarray data for gene expression at 19 developmental points during $B$. mori metamorphosis (unpublished data), we found that 42 of $55 \mathrm{JH}$-related genes were expressed during at least one developmental point (Figure 5). Among the genes involved in the MVA pathway of JH biosynthesis the majority were highly expressed during the pupa-adult transition, with the exception of $M e v K$, whose expression was detected in the female on the fourth day of the fifth larval instar (V4). In particular, Acat and IPPI displayed a high expression in males, whereas FPPS1 and $F P P S 3$ were highly expressed in females. Among the genes involved in the isoprenoid branch of JH biosynthesis, only ALDH-ll, Cyp $15 \mathrm{Cl}$, and several copies of JHAMT and $F A M e T$ were detected during metamorphosis, and they were highly expressed before wandering and during the pupa-adult transition (Figure 5). In particular, FAMeT-l2 and $F A M e T-14$ exhibited a male-specific expression pattern during the pupa-adult transition. Further studies will be re- 


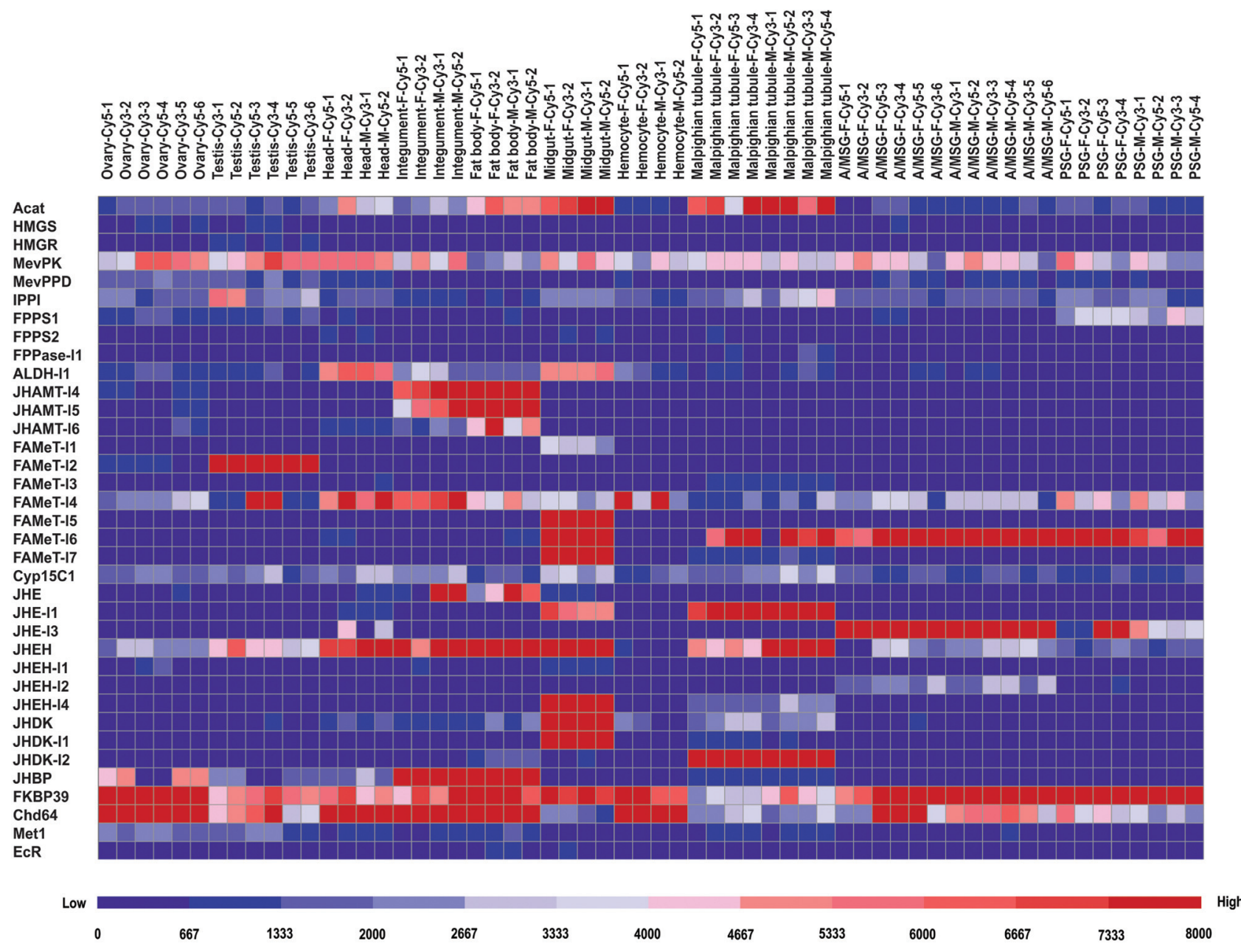

Figure 4 - Expression pattern of JH-related genes in multiple tissues from B. mori larvae. Microarray data of genome-wide gene expression in multiple tissues from B. mori larvae on the third day of the fifth instar were downloaded from the B. mori genome database. Each tissue sample was analyzed with at least two biological repeats. If a JH-related gene was estimated to have an average expression signal intensity of more than 400 in any tissue, it was considered to be expressed in that tissue. F, female; M, male. A/MSG, anterior/median silk gland; PSG, posterior silk gland.

quired to decipher the roles of these two FAMeT genes during the development of $B$. mori males.

Microarray data of gene expression during B. mori metamorphosis also revealed that among the genes involved in JH metabolism, JHE and JHE-ll were highly expressed during pupation. JHEH, JHEH-l2, JHEH-l4, $J H E H-l 5, J H D K-l 1, J H D K$, and JHDK-l2 were mainly expressed before wandering. JHEH-ll and JHEH-l3 displayed a high expression in females during pupa-adult transition. Among the genes involved in JH signaling, $J H B P, F K B P 39$, Chd64, and Met 1 exhibited ubiquitous expression during $B$. mori metamorphosis. Met 2 expression was detected only in males during pupa-adult transition, and $E_{c} R$ and $U S P$ were highly expressed before wandering. $K r-h 1$ presented a high expression during the late stage of the pupa-adult transition.

Because $\mathrm{JH}$ biosynthesis and $\mathrm{JH}$ metabolism are initiated during larval feeding and molting, respectively, we used real-time quantitative RT-PCR experiments to further check the consistency between JH titer and the expression profiles of several JH-related genes with multiple copies during B. mori larval growth. As shown in Figure 6, FPPS1 expression was highly during $B$. mori larval molting and gradually decreased during larval feeding, which was consistent with the changes in JH titer. Interestingly, FPPSI expression at the beginning of the feeding stages was higher in the fifth instar larvae than in the fourth instar larvae. Several copies of the genes involved in the isoprenoid branch of JH biosynthesis, including JHAMT-l3, JHAMT14, FAMeT-l2, and FAMeT-l5, exhibited a high expression during larval feeding and subsequently decreased during larval molting, also indicating consistency with the changes in the JH titer. Moreover, four copies of the genes involved in $\mathrm{JH}$ metabolism, including JHE-l1, JHE-l3, JHDK, JHDK-l1, JHDK-l2, JHEH-l1, JHEH-l3, and JHEH-l5, showed an increased expression during larval feeding and were obviously decreased during larval molting. The ex- 


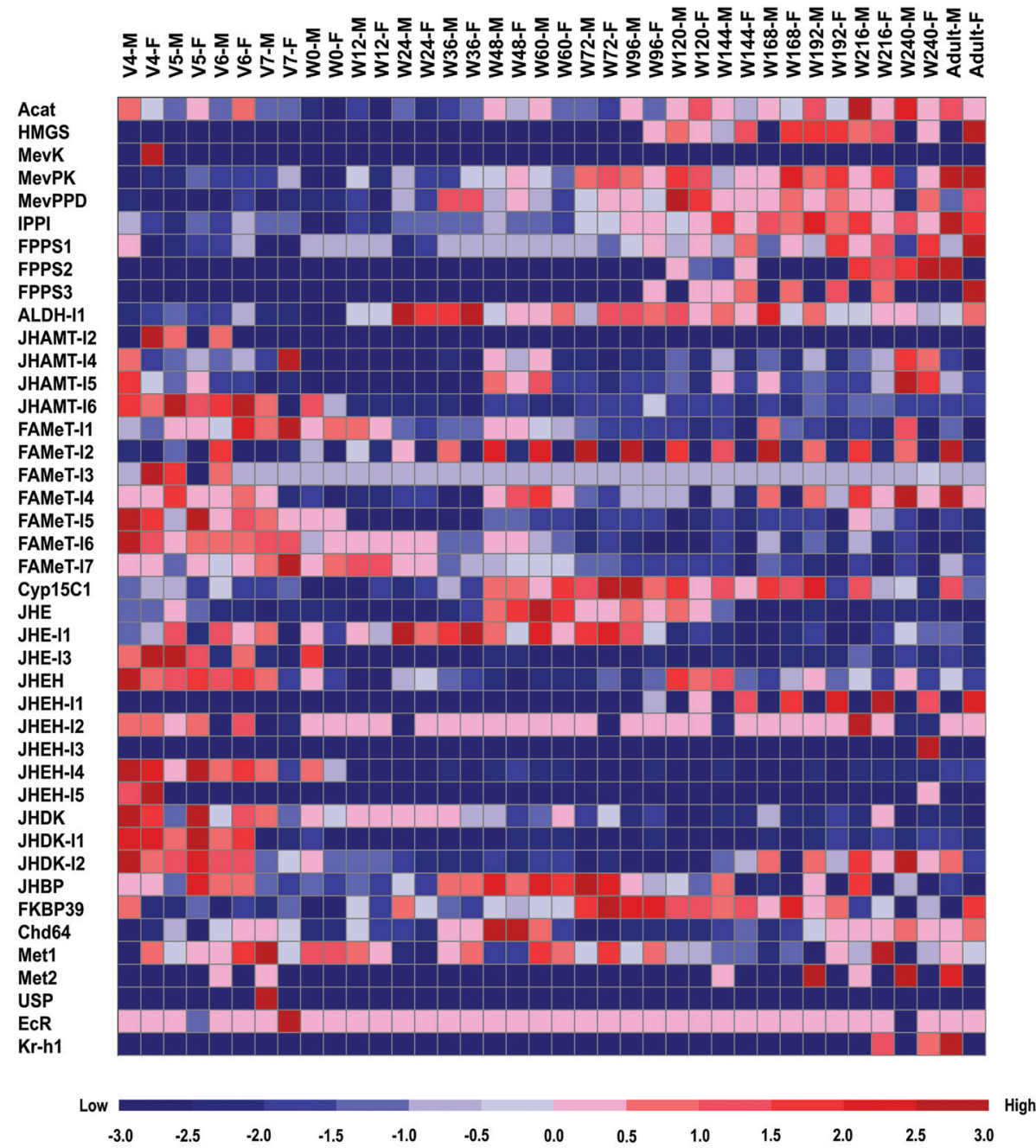

Figure 5 - Expression profiles of JH-related genes during metamorphosis in B. mori. The developmental expression profiles of JH-related genes for $B$. mori were analyzed using microarray data of genome-wide gene expressions at 19 time points during $B$. mori metamorphosis. The ratio was calculated by comparing the signal intensity of the mRNA expression level for each JH-related gene at each time point to that in the common reference of larvae on the third day of the fifth instar in B. mori. Subsequently, the expression change of each JH-related gene was evaluated by this expression ratio and visualized using the GeneCluster2.0 program. Plus and minus denote up- and down-regulation, respectively.

pression of these genes was also consistent with the change in JH titer during B. mori larval feeding and molting.

\section{Discussion}

$\mathrm{JH}$ plays key roles in the regulation of various aspects of insect growth and development. Previous studies have reported the genome-wide identification of genes involved in JH biosynthesis, metabolic degradation, or signaling in several insect species, such as the JH biosynthesis-related genes in B. mori (Kinjoh et al., 2007; Xia et al., 2008), JHEs in A. aegypti (Bai et al., 2007), and JHEHs in T. castaneum (Seino et al., 2010). To understand comprehensively the evolutionary conservation and variation of JHrelated genes in insects, we systematically identified and compared JH-related genes in B. mori and five other insect species whose whole genomes have been sequenced, in- cluding the recently completed $D$. plexippus (Lepidoptera) genome sequence.

The developmental changes in JH titer in insects are mainly controlled by the processes of biosynthesis in the $\mathrm{CA}$ and metabolic degradation in the targeting tissues. Here, we observed an evolutionary divergence of the genes involved in $\mathrm{JH}$ biosynthesis among $B$. mori and other insects. First, the genes related to the upstream seven steps of the MVA pathway of JH biosynthesis, which is responsible for producing IPP as FPP precursor, all existed as a single copy and displayed a rigorous orthologous relationship among the analyzed insects (Table 2 and Table S2), indicating that the process of IPP production was conserved during insect evolution. Second, the FPPS gene involved in the last step of the MVA pathway (production of JH precursor FPP) and all identified genes participating in the isoprenoid branch for producing JH showed a variation in copy num- 

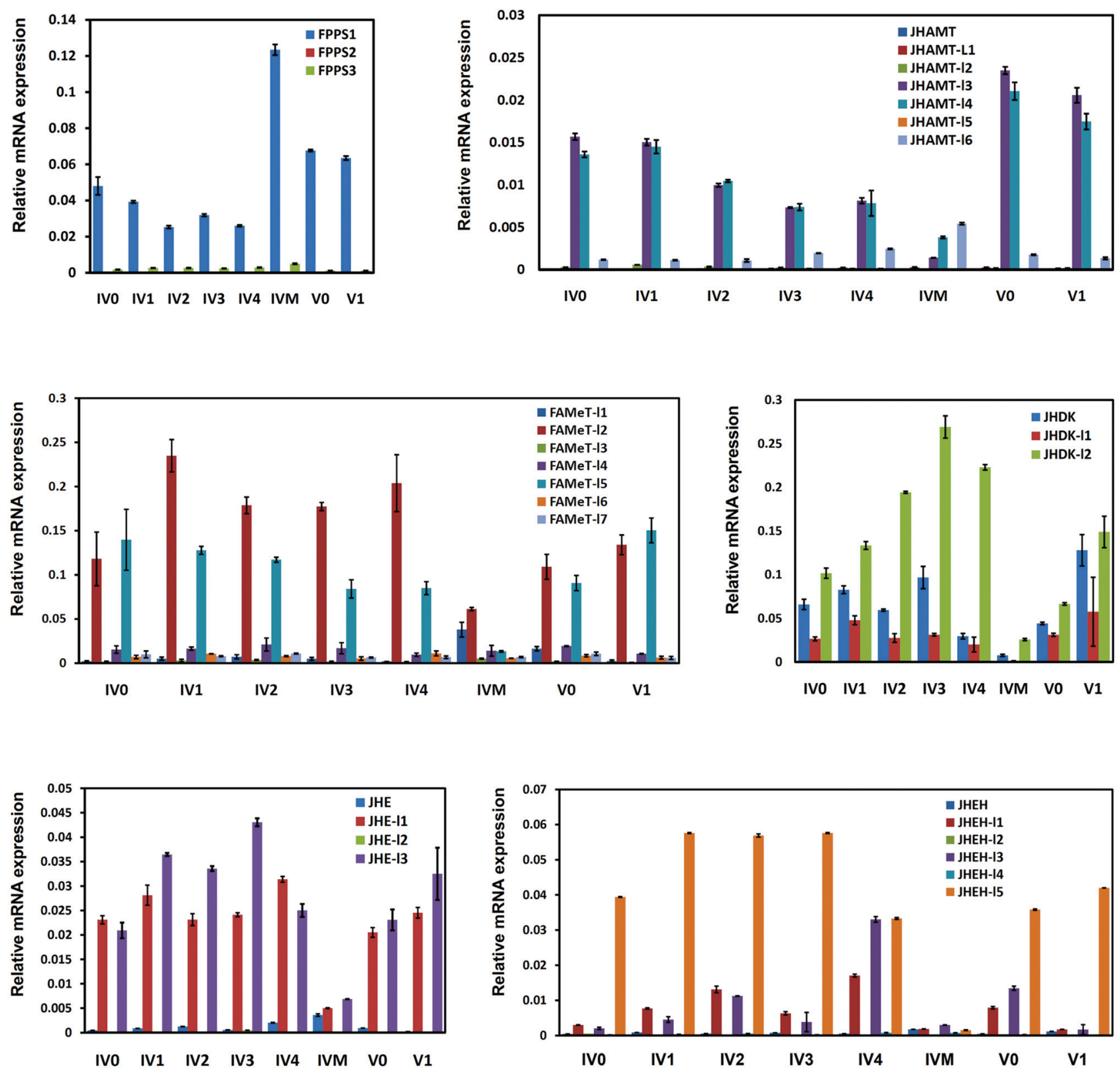

Figure 6 - Expression patterns of JH-related genes with multiple copies during larval growth and molting in B. mori. Quantitative analysis of the gene expression of JH-related genes with multiple copies during larval growth and molting in $B$. mori was performed by real-time RT-PCR. The $B$. mori ribosomal protein L3 (RpL3) gene was used as an internal control. IV0, immediately after completion of the third larval molting and the beginning of feeding in the fourth larval instar; IV1, one day after feeding in the fourth larval instar; IV2, two days after feeding in the fourth larval instar; IV3, three days after feeding in the fourth larval instar; IV4, four days after feeding in the fourth larval instar; IVM, the fourth larval molting; V0, immediately after completion of the fourth larval molting and the beginning of feeding in the fifth larval instar; V1, one day after feeding in the fifth larval instar.

ber or gene class among B. mori and other insects (Table 2 and Table S2), which may be a consequence of gene duplication. In fact, JH types also varied between Lepidoptera and other insect orders, and more types of $\mathrm{JH}$ variants are present in Lepidoptera (Minakuchi et al., 2006; Riddiford, 2008; Goodman and Cusson, 2012; Daimon and Shinoda, 2013; Jindra et al., 2013). Thus, we speculate that the copy variation among the genes involved in the FPP production from IPP and in the isoprenoid branch may reflect the functional divergence of the associated gene products, and is likely linked to the diversity of JH types among insects. Our comparison raised two additional issues regarding the relationship between the variations in JH-related genes and in $\mathrm{JH}$ types. One issue is that FPPS genes from Lepidoptera may have undergone a species-specific divergence (Figure 2), which agrees with previous findings (Kinjoh et al., 2007). Therefore, we hypothesize that FPPS-mediated enzymatic reactions may be the first step leading to the diversity of JH types among insects. Undoubtedly, whether all the predicted FPPS genes are authentically involved in $\mathrm{JH}$ 
biosynthesis needs to be investigated by more enzymatic experiments. However, JHAMT and FAMeT, two enzymes involved in the methyl esterification of FA, were predicted in B. mori and other insects (Table 2 and Table S2). Previous studies in $D$. melanogaster have reported that FAMeT was likely not involved in JH biosynthesis (Burtenshaw et al., 2008; Zhang et al., 2010). However, another analysis speculated that FAMeT-mediated methyl esterification should exist in insects and crustaceans (Hui et al., 2010). Undoubtedly, more evidence is required to resolve the controversial functions of FAMeTs in JH biosynthesis. Furthermore, Cyp15C1 and Cyp15A1 were confirmed to be involved in two routes of FA epoxidation (Daimon and Shinoda, 2013). However, several surveyed insects do not contain either Cyp15C1 or Cyp15A1 (Table 2 and Table S2). Thus, additional experiments are needed to address whether both epoxidation routes occur in insects with no Cyp15C1 or no Cyp15A1 or whether other Cyp genes whose functions have yet to be identified catalyze one or the other route. Finally, all identified genes involved in neuropeptide regulation of JH biosynthesis existed as a single copy, implying that their regulatory mechanism is also conserved among insects.

The metabolic degradation of $\mathrm{JH}$ is catalyzed mainly by JHE, JHEH, and JHDK. Our results showed that each of the genes encoding these three enzymes may have undergone duplication in Lepidoptera (Table 2 and Table S2). In particular, JHDK has multiple copies in Lepidoptera but one copy in the other surveyed insects. Given the existence of more JH types in Lepidoptera, we propose that, as was true of copy variation among several JH biosynthesisrelated genes, the duplication and divergence of JH metabolism-related genes may also be a functionally adaptive evolution to the diversity of $\mathrm{JH}$ types in insects.

The nature of $\mathrm{JH}$ signaling has been the subject of an increasing number of experimental studies. To date, it has been confirmed that seven genes, namely, JHBP, FKBP39, Chd64, EcR, USP, Met, and $\mathrm{Kr}$ - $h 1$, are implicated in JH signaling (Li et al., 2007; Suzuki et al., 2011: Jindra et al., 2013). Except for Met, each of the other genes existed as a single copy in B. mori and other surveyed insects (Table 2 and Table S2). The Met protein is a bHLH-PAS transcription factor and has been characterized as a $\mathrm{JH}$ receptor in insects (Wilson and Fabian, 1986; Jindra et al., 2013). Unlike the single copy of the Met gene in A. gambiae, A. mellifera, and $T$. castaneum, two copies of the Met gene have been identified previously in other insects, namely, Met and Gce in Drosophila (Baumann et al., 2010b) and Met1 and Met2 in B. mori (Li et al., 2010; Guo et al., 2012; Kayukawa et al., 2012). Our analysis also identified two copies (Met-l1 and Met-l2) of the Met gene in two other lepidopterans, $D$. plexippus and H. melpomene. Although current evidence is not sufficient to elucidate why Met was duplicated in these insects, two copies of the Met gene have been demonstrated to cooperatively modulate $\mathrm{JH}$ signaling via a protein- protein interaction in D. melanogaster (Baumann et al., 2010a; Abdou et al., 2011) and in B. mori (Guo et al., 2012; Kayukawa et al., 2012). Together with the findings from the phylogenetic tree of insect Met genes (Figure 3) and the functional redundancy of two copies of the $\mathrm{Met}$ gene, we propose that the $\mathrm{JH}$ signaling cascade is also evolutionarily conserved across insects.

Given that multiple copies were predicted for several genes involved in JH biosynthesis and metabolism, it was important to determine whether these copies were functional. Our microarray data and quantitative RT-PCR experiments in B. mori showed that some copies of the JHrelated genes exhibited moderate expression in the head (mainly containing CA, CC, and brain) or in a dynamic manner that correlated with the temporal changes in JH titer, such as FPPS1, FPPS2, JHAMT-13, JHAMT-14, JHE$11, J H E H-l 5, J H D K$, and JHDK-l2. Previous studies have confirmed the enzymatic activities or physiological functions of several JH-related genes in B. mori, such as JHAMT (Shinoda and Itoyama, 2003), JHE (Tan et al., 2005), JHEH (Seino et al., 2010), and JHDK (Li et al., 2005). One copy of the JHAMT gene has also been verified to be activated functionally in $\mathrm{JH}$ biosynthesis in $T$. castaneum (Minakuchi and Riddiford, 2008). Furthermore, most enzyme-encoding genes with multiple copies appear to be expressed in other larval tissues excluding head (mainly containing $\mathrm{CA}, \mathrm{CC}$, and brain), during developmental stages with no JH activities, or in one or other sex (Figures 4, 5 and 6). These observations indicate that the copies of the JH-related genes may play roles in other physiological processes. Nevertheless, further enzymatic activity assays are undoubtedly required to assess whether multiple copies of the JH-related genes encode authentic enzymes involved in JH biosynthesis and metabolism or encode proteins involved in other physiological processes.

In summary, we first performed a systematic identification of the genes involved in JH biosynthesis, metabolism, and signaling in insects, including two lepidopterans (B. mori and D. plexippus), two dipterans (D. melanogaster and A. gambiae), one hymenopteran (A. mellifera), and one coleopteran (T. castaneum). A comparative analysis concluded that the early steps of the MVA pathway and neuropeptide regulation of $\mathrm{JH}$ biosynthesis, as well as $\mathrm{JH}$ signaling, are apparently conserved among $B$. mori and other surveyed insects. However, most genes involved in the last step of the MVA pathway and the isoprenoid branch of $\mathrm{JH}$ biosynthesis, as well as JH metabolism, seem to have undergone duplication, resulting in multiple copies in Lepidoptera. This duplication may be functionally and evolutionarily relevant to the variation of $\mathrm{JH}$ types among Lepidoptera and other insect species. Although some copies of several JH-related multi-copy genes show a specific spatio-temporal expression correlated to JH activity in $B$. mori, it remains to be confirmed whether their enzymatic activities are associated with $\mathrm{JH}$ biosynthesis and metabo- 
lism. Taken together, the results of our analysis provide new clues for understanding the genetic basis of JH biosynthesis, metabolism, and signaling in insects.

\section{Acknowledgments}

This work was supported by grants from the National Basic Research Program of China (No. 2012CB114600), the National Hi-Tech Research and Development Program of China (No. 2011AA100306), the National Natural Science Foundation of China (No. 31172267 and No. 31272503), and the Municipal Natural Science Foundation of Chongqing (No. cstc2012jjA80023).

\section{References}

Abdou MA, He Q, Wen D, Zyaan O, Wang J, Xu J, Baumann AA, Joseph J, Wilson TG, Li S, et al. (2011) Drosophila Met and Gce are partially redundant in transducing juvenile hormone action. Insect Biochem Mol Biol 41:938-945.

Bai H, Ramaseshadri P and Palli SR (2007) Identification and characterization of juvenile hormone esterase gene from the yellow fever mosquito, Aedes aegypti. Insect Biochem Mol Biol 37:829-837.

Baumann A, Barry J, Wang S, Fujiwara Y and Wilson TG (2010a) Paralogous genes involved in juvenile hormone action in Drosophila melanogaster. Genetics 185:1327-1336.

Baumann A, Fujiwara Y and Wilson TG (2010b) Evolutionary divergence of the paralogs Methoprene tolerant (Met) and germ cell expressed (gce) within the genus Drosophila. J Insect Physiol 56:1445-1455.

Bogus M and Scheller K (1996) Allatotropin released by the brain controls larval molting in Galleria mellonella by affecting juvenile hormone synthesis. Int J Dev Biol 40:205-210.

Bownes M and Rembold H (1987) The titre of juvenile hormone during the pupal and adult stages of the life cycle of Drosophila melanogaster. Eur J Biochem 164:709-712.

Burtenshaw SM, Su PP, Zhang JR, Tobe SS, Dayton L and Bendena WG (2008) A putative farnesoic acid O-methyltransferase (FAMeT) orthologue in Drosophila melanogaster (CG10527): Relationship to juvenile hormone biosynthesis? Peptides 29:242-251.

Daimon T and Shinoda T (2013) Function, diversity, and application of insect juvenile hormone epoxidases (CYP15). Biotechnol Appl Biochem 60:82-91.

Daimon T, Kozaki T, Niwa R, Kobayashi I, Furuta K, Namiki T, Uchino K, Banno Y, Katsuma S, Tamura T, et al. (2012) Precocious metamorphosis in the juvenile hormonedeficient mutant of the silkworm, Bombyx mori. PLoS Genetics 8:e1002486.

Dubrovsky EB (2005) Hormonal cross talk in insect development. Trends Endocrinol Metab 16:6-11.

Furuta K, Ichikawa A, Murata M, Kuwano E, Shinoda T and Shiotsuki T (2013) Determination by LC-MS of juvenile hormone titers in hemolymph of the silkworm, Bombyx mori. Biosci Biotechnol Biochem 77:988-991.

Futahashi R and Fujiwara H (2008) Juvenile hormone regulates butterfly larval pattern switches. Science 319:1061.

Goodman WG and Cusson M (2012) The Juvenile Hormones. In: Gilbert LI (ed) Insect Endocrinology. Academic Press, London, pp 310-365.
Guo EE, He QY, Liu SM, Tian L, Sheng ZT, Peng Q, Guan JM, Shi MA, Li K, Gilbert LI, et al. (2012) METis required for the maximal action of 20-Hydroxyecdysone during Bombyx metamorphosis. PLoS One 7:e53256.

Heliconius Genome C (2012) Butterfly genome reveals promiscuous exchange of mimicry adaptations among species. Nature 487:94-98.

Hirai M, Kamimura M, Kikuchi K, Yasukochi Y, Kiuchi M, Shinoda T and Shiotsuki T (2002) cDNA cloning and characterization of Bombyx mori juvenile hormone esterase: An inducible gene by the imidazole insect growth regulator KK-42. Insect Biochem Mol Biol 32:627-635.

Horodyski FM, Verlinden H, Filkin N, Vandersmissen HP, Fleury C, Reynolds SE, Kai ZP and Broeck JV (2011) Isolation and functional characterization of an allatotropin receptor from Manduca sexta. Insect Biochem Mol Biol 41:804-814.

Hua-Jun Y, Fang Z, Awquib S, Malik FA, Roy B, Xing-Hua L, Jia-Biao H, Chun-Guang S, Niu YS and Yun-Gen M (2011) Expression pattern of enzymes related to juvenile hormone metabolism in the silkworm, Bombyx mori L. Molecular Biol Reports 38:4337-4342.

Huang LL, Cheng TC, Xu PZ, Cheng DJ, Fang T and Xia QY (2009) A genome-wide survey for host response of silkworm, Bombyx mori during pathogen Bacillus bombyseptieus infection. PLoS One 4:e8098.

Hui JH, Hayward A, Bendena WG, Takahashi T and Tobe SS (2010) Evolution and functional divergence of enzymes involved in sesquiterpenoid hormone biosynthesis in crustaceans and insects. Peptides 31:451-455.

Jindra M, Palli SR and Riddiford LM (2013) The juvenile hormone signaling pathway in insect development. Annu Rev Entomol 58:181-204.

Kataoka H, Toschi A, Li JP, Carney RL, Schooley DA and Kramer SJ (1989) Identification of an allatotropin from adult Manduca sexta. Science 243:1481-1483.

Kayukawa T, Minakuchi C, Namiki T, Togawa T, Yoshiyama M, Kamimura M, Mita K, Imanishi S, Kiuchi M, Ishikawa Y, et al. (2012) Transcriptional regulation of juvenile hormonemediated induction of Kruppel homolog 1, a repressor of insect metamorphosis. Proc Natl Acad Sci USA 109:1172911734.

Kinjoh T, Kaneko Y, Itoyama K, Mita K, Hiruma K and Shinoda $\mathrm{T}$ (2007) Control of juvenile hormone biosynthesis in Bombyx mori: Cloning of the enzymes in the mevalonate pathway and assessment of their developmental expression in the corpora allata. Insect Biochem Mol Biol 37:808-818.

Kramer SJ, Toschi A, Miller CA, Kataoka H, Quistad GB, Li JP, Carney RL and Schooley DA (1991) Identification of an allatostatin from the tobacco hornworm Manduca sexta. Proc Natl Acad Sci USA 88:9458-9462.

Li S, Jiang R and Cao M (2004) Metabolism of juvenile hormone. Acta Entomol Sin 47:389-393.

Li S, Zhang QR, Xu WH and Schooley DA (2005) Juvenile hormone diol kinase, a calcium-binding protein with kinase activity, from the silkworm, Bombyx mori. Insect Biochem Mol Biol 35:1235-1248.

Li Y, Zhang Z, Robinson GE and Palli SR (2007) Identification and characterization of a juvenile hormone response element and its binding proteins. J Biol Chem 282:37605-37617.

Li Z, Cheng D, Wei L, Zhao P, Shu X, Tang L, Xiang Z and Xia Q (2010) The silkworm homolog of Methoprene-tolerant 
(Met) gene reveals sequence conservation but function divergence. Insect Sci 17:313-324.

Maxwell RA, Welch WH and Schooley DA (2002) Juvenile hormone diol kinase. I. Purification, characterization, and substrate specificity of juvenile hormone-selective diol kinase from Manduca sexta. J Biol Chem 277:21874-21881.

Mayoral JG, Nouzova M, Navare A and Noriega FG (2009) NADP+-dependent farnesol dehydrogenase, a corpora allata enzyme involved in juvenile hormone synthesis. Proc Natl Acad Sci USA 106:21091-21096.

Minakuchi C, Namiki T, Yoshiyama M and Shinoda T (2008) RNAi-mediated knockdown of juvenile hormone acid O-methyltransferase gene causes precocious metamorphosis in the red flour beetle Tribolium castaneum. FEBS J 275:2919-2931.

Minakuchi C and Riddiford L (2006) Insect juvenile hormone action as a potential target of pest management. J Pesticide Sci 31:77-84

Munoz-Torres MC, Reese JT, Childers CP, Bennett AK, Sundaram JP, Childs KL, Anzola JM, Milshina N and Elsik CG (2011) Hymenoptera Genome Database: Integrated community resources for insect species of the order Hymenoptera. Nucleic Acids Res 39:D658-D662.

Noriega FG (2004) Nutritional regulation of JH synthesis: A mechanism to control reproductive maturation in mosquitoes? Insect Biochem Mol Biol 34:687-693.

Noriega FG, Ribeiro JM, Koener JF, Valenzuela JG, HernandezMartinez S, Pham VM and Feyereisen R (2006) Comparative genomics of insect juvenile hormone biosynthesis. Insect Biochem Mol Biol 36:366-374.

Nyati P, Nouzova M, Rivera-Perez C, Clifton ME, Mayoral JG and Noriega FG (2013) Farnesyl phosphatase, a corpora allata enzyme involved in juvenile hormone biosynthesis in Aedes aegypti. PLoS One 8:e71967.

Park C, Hwang JS, Kang SW and Lee BH (2002) Molecular characterization of a cDNA from the silk moth Bombyx mori encoding Manduca sexta allatotropin peptide. Zool Sci 19:287-292.

Reich M, Ohm K, Angelo M, Tamayo P and Mesirov JP (2004) GeneCluster 2.0: An advanced toolset for bioarray analysis. Bioinformatics 20:1797-1798.

Riddiford LM (1994) Cellular and molecular action of juvenile hormone 1 General considerations and premetamorphic actions. Adv Insect Physiol 24:213-274.

Riddiford LM (2008) Juvenile hormone action: A 2007 perspective. J Insect Physiol 54:895-901.

Riddiford LM (2012) How does juvenile hormone control insect metamorphosis and reproduction? Gen Comp Endocrinol 179:477-484.

Rivera-Perez C, Nouzova M, Clifton ME, Garcia EM, LeBlanc E and Noriega FG (2013) Aldehyde dehydrogenase 3 converts farnesal into farnesoic acid in the corpora allata of mosquitoes. Insect Biochem Mol Biol 43:675-682.

Roller L, Yamanaka N, Watanabe K, Daubnerova I, Zitnan D, Kataoka H and Tanaka Y (2008) The unique evolution of neuropeptide genes in the silkworm Bombyx mori. Insect Biochem Mol Biol 38:1147-1157.

Sakurai S and Niimi S (1997) Development changes in juvenile hormone and juvenile hormone acid titers in the hemolymph and in-vitro juvenile hormone synthesis by corpora allata of the silkworm, Bombyx mori. J Insect Physiol 43:875-884.
Secher T, Lenz C, Cazzamali G, Sorensen G, Williamson M, Hansen GN, Svane P and Grimmelikhuijzen CJ (2001) Molecular cloning of a functional allatostatin gut/brain receptor and an allatostatin preprohormone from the silkworm Bombyx mori. J Biol Chem 276:47052-47060.

Seino A, Ogura T, Tsubota T, Shimomura M, Nakakura T, Tan A, Mita K, Shinoda T, Nakagawa Y and Shiotsuki T (2010) Characterization of juvenile hormone epoxide hydrolase and related genes in the larval development of the silkworm Bombyx mori. Biosci Biotechnol Biochem 74:1421-1429.

Shinoda T and Itoyama K (2003) Juvenile hormone acid methyltransferase: A key regulatory enzyme for insect metamorphosis. Proc Natl Acad Sci USA 100:11986-11991.

Stay B (2000) A review of the role of neurosecretion in the control of juvenile hormone synthesis: A tribute to Berta Scharrer. Insect Biochem Mol Biol 30:653-662.

Suzuki R, Fujimoto Z, Shiotsuki T, Tsuchiya W, Momma M, Tase A, Miyazawa M and Yamazaki T (2011) Structural mechanism of JH delivery in hemolymph by JHBP of silkworm, Bombyx mori. Sci Rep 1:133.

Tamura K, Dudley J, Nei M and Kumar S (2007) MEGA4: Molecular Evolutionary Genetics Analysis (MEGA) software version 4.0. Mol Biol Evol 24:1596-1599.

Tan A, Tanaka H, Tamura T and Shiotsuki T (2005) Precocious metamorphosis in transgenic silkworms overexpressing juvenile hormone esterase. Proc Natl Acad Sci USA 102:11751-11756.

Thompson JD, Gibson TJ, Plewniak F, Jeanmougin F and Higgins DG (1997) The CLUSTAL X windows interface: Flexible strategies for multiple sequence alignment aided by quality analysis tools. Nucleic Acids Res 25:4876-4882.

Wang GH, Liu C, Xia QY, Zha XF, Chen J and Jiang L (2008) Cathepsin $\mathrm{B}$ protease is required for metamorphism in silkworm, Bombyx mori. Insect Sci 15:201-208.

Wilson TG and Fabian J (1986) A Drosophila melanogaster mutant resistant to a chemical analog of juvenile hormone. Dev Biol 118:190-201.

Wyatt G and Davey K (1996) Cellular and molecular actions of juvenile hormone. II. Roles of juvenile hormone in adult insects. Advances in Insect Physiology 26:1-155.

Xia Q, Cheng D, Duan J, Wang G, Cheng T, Zha X, Liu C, Zhao P, Dai F, Zhang Z, et al. (2007) Microarray-based gene expression profiles in multiple tissues of the domesticated silkworm, Bombyx mori. Genome Biol 8:R162.

Xia QY, Wang J, Zhou ZY, Li RQ, Fan W, Cheng DJ, Cheng TC, Qin JJ, Duan J, Xu HF, et al. (2008) The genome of a lepidopteran model insect, the silkworm Bombyx mori. Insect Biochem Mol Biol 38:1036-1045.

Yamamoto K, Nohata J, Kadono-Okuda K, Narukawa J, Sasanuma M, Sasanuma S, Minami H, Shimomura M, Suetsugu Y, Banno Y, et al. (2008) A BAC-based integrated linkage map of the silkworm Bombyx mori. Genome Biol 9:R21.

Yamamoto R, Bai H, Dolezal AG, Amdam G and Tatar M (2013) Juvenile hormone regulation of Drosophila aging. BMC Biology 11:e85.

Yamanaka N, Yamamoto S, Zitnan D, Watanabe K, Kawada T, Satake H, Kaneko Y, Hiruma K, Tanaka Y, Shinoda T, et al. (2008) Neuropeptide receptor transcriptome reveals unidentified neuroendocrine pathways. PLoS One 3:e3048.

Zhan S and Reppert SM (2013) MonarchBase: The monarch butterfly genome database. Nucleic Acids Res 41:D758-763. 
Zhang H, Tian L, Tobe S, Xiong Y, Wang S, Lin X, Liu Y, Bendena W, Li S and Zhang YQ (2010) Drosophila CG10527 mutants are resistant to juvenile hormone and its analog methoprene. Biochem Biophys Res Commun 401:182-187.

Zhang QR, Xu WH, Chen FS and Li S (2005) Molecular and biochemical characterization of juvenile hormone epoxide hydrolase from the silkworm, Bombyx mori. IInsect Biochem Mol Biol 35:153-164.

Zou Z, Saha TT, Roy S, Shin SW, Backman TW, Girke T, White KP and Raikhel AS (2013) Juvenile hormone and its receptor, methoprene-tolerant, control the dynamics of mosquito gene expression. Proc Natl Acad Sci USA 110:E2173E2181.

\section{Internet Resources}

SMART program, http://smart.embl-heidelberg.de/.

Microarray data in Bombyx mori genome database, http://www.silkdb.org/microarray/download.html.

\section{Supplementary Material}

The following online material is available for this article:

Table S1 - RT-PCR Primers used in this study.

Table S2 - JH-related genes in the other surveyed insects.

Figure S1 - Summary of insect JH-related pathways.

Figure S2 - Phylogenetic tree of the JHAMT and FAMeT genes.

Figure S3 - Phylogenetic tree of the JHE, JHEH, and JHDK genes.

This material is available as part of the online article from http://www.scielo.br/gmb.

Associate Editor: Houtan Noushmehr

License information: This is an open-access article distributed under the terms of the Creative Commons Attribution License, which permits unrestricted use, distribution, and reproduction in any medium, provided the original work is properly cited. 